\title{
The XMM-Newton view of stellar coronae: Flare heating in the coronae of HR 1099*
}

\author{
M. Audard ${ }^{1}$, M. Güdel ${ }^{1}$, and R. Mewe ${ }^{2}$ \\ 1 Paul Scherrer Institut, Würenlingen \& Villigen, 5232 Villigen PSI, Switzerland \\ 2 SRON Laboratory for Space Research, Sorbonnelaan 2, 3584 CA Utrecht, The Netherlands
}

Received 2 October 2000 / Accepted 30 October 2000

\begin{abstract}
The RS CVn system HR 1099 was observed by XMM-Newton for a time span of 25 days during the commissioning phase. Rotational modulation in the RGS light curve has been detected with maximum flux when the active K1 IV star is in front. Following a quiescent phase of emission, the rise and peak of a large flare were observed. Time-dependent spectroscopy has been performed to derive elemental abundances and their variations during the various stages of the flare. Emission measure distributions have been reconstructed. Two different components are detected: a very hot plasma (up to $100 \mathrm{MK}$ ) that evolves rapidly, and a stable quiescent plasma. Low first-ionization-potential elemental (such as $\mathrm{Fe}$ and $\mathrm{Si}$ ) abundances increase significantly during the flare, while the abundance of the high-FIP element Ne stays constant at the quiescent value. We report direct detection of a flux increase in the FexxIV X-ray lines during a stellar flare.
\end{abstract}

Key words. stars: abundances - stars: activity - stars: coronae - stars: flare - stars: individual: HR 1099 X-rays: stars

\section{Introduction}

Flares are frequently observed on magnetically active stars from the radio to the $\mathrm{X}$-ray regime. Sudden reconfiguration of the magnetic field through reconnection is believed to release magnetic energy. In a standard model, coronal electrons are accelerated; part of these electrons collide in the dense chromospheric layer of the stellar atmosphere, producing non-thermal hard X-ray emission (e.g., Dennis 1985). Energy dissipation in the dense layers produces heating of chromospheric material: neutral or already partially ionized material is further ionized. The pressure increases and coronal loops become filled with hot material (chromospheric evaporation; see Antonucci et al. 1984). Radiative cooling occurs mainly through various boundbound electronic transitions of elements such as Fe, Si, S, $\mathrm{Ne}, \mathrm{C}, \mathrm{N}, \mathrm{O}, \mathrm{Mg}$, etc., and continuum emission composed of a superposition of two-photon processes, free-bound emission and thermal bremsstrahlung (free-free emission). The emission lines allow us to derive several plasma properties, such as the distribution of emission measure $(E M)$

Send offprint requests to: M. Audard, e-mail: audard@astro.phys.ethz.ch

* Based on observations obtained with XMM-Newton, an ESA science mission with instruments and contributions directly funded by ESA Member States and the USA (NASA). as a function of plasma temperature, elemental abundances, or average electron densities (see Mewe 1999).

Flares are of central importance to coronal heating. Large flares can display very high temperatures (e.g., Pallavicini \& Tagliaferri 1998). Tsuboi et al. (1998) report temperatures of at least $100 \mathrm{MK}$ in a flare on the weak-lined T Tau star V773 Tau. Güdel et al. (1999) showed that the flare EM distribution of the RS CVn binary UX Ari evolved to temperatures of 50 to $100 \mathrm{MK}$ and was accompanied by individual elemental abundance variations. Such variations were already suggested in previous observations, although elemental abundances were often linked together to provide best fits with an "average" metal abundance (e.g., Ottmann \& Schmitt 1996; Favata et al. 2000). Data with low spectral resolution could not disentangle the emission lines from the underlying continuum, which led to some uncertainties about metaldeficient coronal abundances in stars compared to their photospheric values (Schmitt et al. 1996). Furthermore, on some stars, elements with low first ionization potentials (FIP) are found to be enhanced with respect to their photospheric abundances (the FIP effect), while on others they are not (e.g., Drake et al. 1995, 1997).

HR 1099 (V711 Tauri; HD 22468) is a binary system of the RS CVn class. The system consists of K1 IV + G5 IV stars that are tidally locked with a period of 2.84 days and an inclination of $i=33^{\circ}$ (Bopp \& Fekel 1976; 


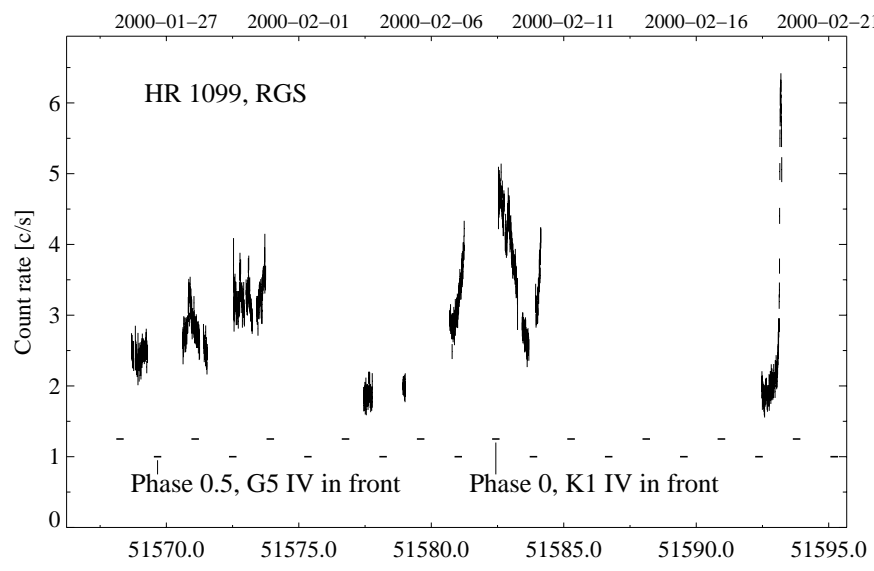

Fig. 1. Total 1st and 2nd order RGS2 light curve of HR 1099. Between MJD 51580.702 - 51581.244, RGS1 data were added, because RGS2 was not observing. The ephemeris of Vogt et al. (1999) has been used

Fekel 1983). At a distance of 28.97 pc (Perryman et al. 1997), it is one of the brightest members of its class. HR 1099 has been extensively studied in the optical, the ultra-violet and the radio, while its extreme ultra-violet (EUV) and X-ray emission was analyzed in the context of surveys of coronal emission from RS CVn systems (Majer et al. 1986; Pasquini et al. 1989; Schmitt et al. 1990; Griffiths \& Jordan 1998). Possible detection of rotational modulation in the EUV light curve of HR 1099 was reported by Drake et al. (1994), with a minimum flux occurring near the phase when the G5 star is in front $(\phi=0.5)$, consistent with a previously reported correlation between binary phase and X-ray intensity by Agrawal \& Vaidya (1988). Recently, Ayres et al. (2001) found in Chandra HETG data of HR 1099 that wavelength shifts in the Nex Ly $\alpha$ line are consistent with the orbital motion of the active K1 IV star. In the context of spectroscopy, Drake \& Kashyap (1998) studied the coronal metallicity of HR 1099 . They reported a coronal iron abundance of $[\mathrm{Fe} / \mathrm{H}] \approx-0.4$, consistent with photospheric iron abundance of the $\mathrm{K} 1 \operatorname{star}([\mathrm{Fe} / \mathrm{H}] \approx-0.6$, Randich et al. 1994). However, noting the difference in photospheric abundances between the primary and the secondary, they argued that the measurements of photospheric abundances of RS CVn systems may be incorrect. Brinkman et al. (2001) analyse XMM-Newton RGS data, discussing coronal elemental abundances in HR 1099. They compare coronal elemental enrichment with solar abundance ratios and find and inverse FIP effect for the time-averaged X-ray emission. The present paper complements the Brinkman et al. paper by investigating the variability of the X-ray emission of HR 1099, the variation of the elemental abundances and of the temperature structure during a large flare (Table 1).
Table 1. Observation $\log$ on 2000 February 18/19

\begin{tabular}{lll}
\hline$\alpha[\mathrm{hh}: \mathrm{mm}: \mathrm{ss}]$ & $\delta[\mathrm{dd}: \mathrm{mm}: \mathrm{ss}]$ & PA [dd:mm:ss] \\
\hline $03: 37: 14.7^{\mathrm{a}}$ & $+00: 36: 40.3^{\mathrm{a}}$ & $257: 41: 00.9^{\mathrm{a}}$ \\
\hline Detector & Start [UT] & Stop [UT] \\
\hline RGS1 & $13: 25: 10$ & $05: 25: 15$ \\
RGS2 & $13: 25: 10$ & $05: 25: 18$ \\
MOS1 & $14: 26: 33$ & $05: 26: 00$ \\
MOS2 $_{\text {pn }^{\mathrm{b}}}$ & $14: 26: 35$ & $05: 26: 00$ \\
\hline
\end{tabular}

${ }^{\mathrm{a}}$ Off-axis pointing by $-7^{\prime}$ in dispersion direction.

${ }^{b}$ Source fell on CCD gaps, therefore no useful data.

Table 2. Time intervals for the phase-dependent analysis

\begin{tabular}{llll}
\hline Interval & Start [UT] & Stop [UT] & MJD $[51592.0+]$ \\
\hline Quiescent & $13: 25: 10^{\mathrm{a}}$ & $01: 38: 30$ & $0.55914-1.06840$ \\
Rise part 1 & $01: 38: 30$ & $03: 01: 50$ & $1.06840-1.12627$ \\
Rise part 2 & $03: 01: 50$ & $03: 51: 50$ & $1.12627-1.16100$ \\
Peak & $03: 51: 50$ & $05: 26: 00$ & $1.16100-1.22639$ \\
\hline
\end{tabular}

${ }^{a}$ MOS2 observation begins 1 hour later.

\section{Data reduction and analysis}

HR 1099 was observed during the early commissioning phase of XMM-Newton (Jansen et al. 2001). The satellite carries $5 \mathrm{X}$-ray detectors and an optical telescope: two MOS European Photon Imaging Cameras (EPIC; Turner et al. 2001), one EPIC pn (Strüder et al. 2001), two Reflection Grating Spectrometers (RGS; den Herder et al. 2001) and an Optical Monitor (Mason et al. 2001). HR 1099 was, as the first-light target of the RGS, monitored over a time span of about 25 days (see Fig. 1).

The data were analyzed with the official ESA XMM Science Analysis System (SAS) software, version 4.1, together with the latest calibration files available at the time of the analysis. The metatask RGSPROC 0.73 .3 was used to process the RGS data. Normally, the dispersion angles can be corrected for attitude drifts during the exposure. However, since the full attitude information was not available for these early observations, no correction was applied. Nevertheless, preliminary attitude data showed that the observation was stable during the data acquisition. Spectra were extracted along the dispersion direction using a spatial mask together with a cut in the plane of dispersion angle vs. CCD energy. The RGS response matrix was created with RGSRMFGEN 0.29. The metatask EMPROC 1.8 was used to process the EPIC MOS2 data (the source was placed in the middle of a CCD gap for both EPIC MOS1 and pn). The EPIC MOS2 data were taken in the PRI FULL WIN mode and with the MEDIUM filter, hence they suffered from severe pile-up and optical contamination. Lumb et al. (2000) showed that removing the center of the Point Spread Function, which is heavily 


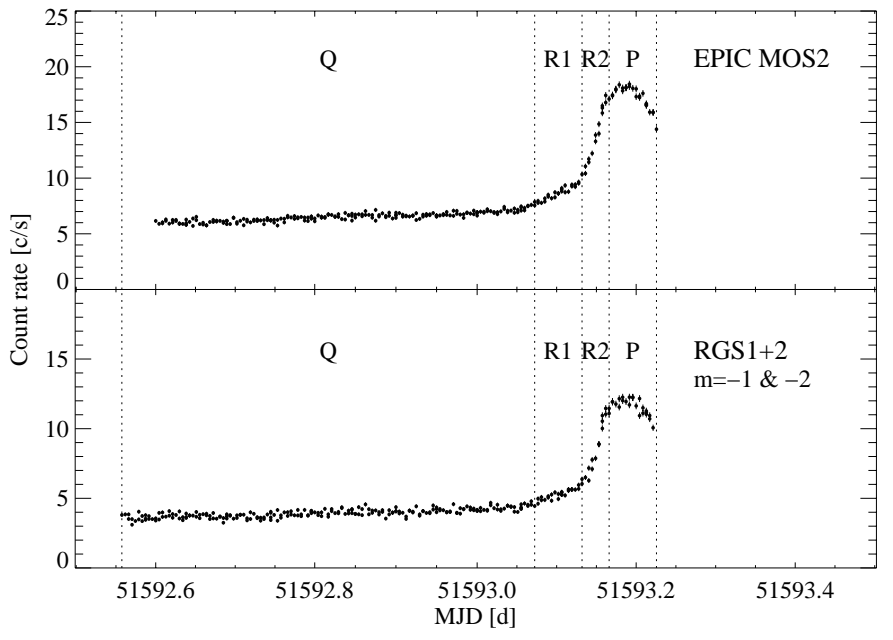

Fig. 2. EPIC MOS2 and coadded RGS1+2 1st and 2nd order light curves with chosen time intervals (Table 2)

piled-up, and extracting source counts from an annulus permits a compromised use of the data, naturally with a reduced count rate. The MOS2 response and ancillary response matrices were created by RMFGEN 1.37 .2 and ARFGEN 1.35, respectively.

Time-dependent spectroscopy was performed for EPIC MOS2 and both RGS instruments. To study the large flare which occurred after MJD 51593.1, we have selected four time intervals, designated "quiescent", "rise part 1", "rise part 2", and "peak" (Table 2). Figure 2 shows the chosen time slices together with the light curves in the analyzed instruments.

\section{Results}

\subsection{Rotational modulation}

The long light curve of HR 1099 (Fig. 1) displays evident variability on short and long time-scales. Additional to the large flare at MJD 51593.1, several other possible smaller flares can be seen (e.g., at $51571 \mathrm{~d}$, and around $51573 \mathrm{~d}$ ). An additional feature of the light curve that we interpret as rotational modulation is seen around MJD 51582. The X-ray flux appears to peak when the active K1 star is in front (phase $\phi=0$ ), while the flux diminishes until it reaches a minimum value when the companion G5 star is in front. On a smaller scale of flux variations, this behaviour is also suggested during earlier phases (MJD 51571.3, 51573.8). The strong variability from one rotation period to the other may suggest that long-term flaring is involved, and that the MJD 51582 emission is rotationally modulated flare emission. Spectral hardness analysis, however, does not show clear signatures of heating and cooling, in contrast to the later flare discussed below. The episode around MJD 51582 may represent general enhanced activity on the more active hemisphere.
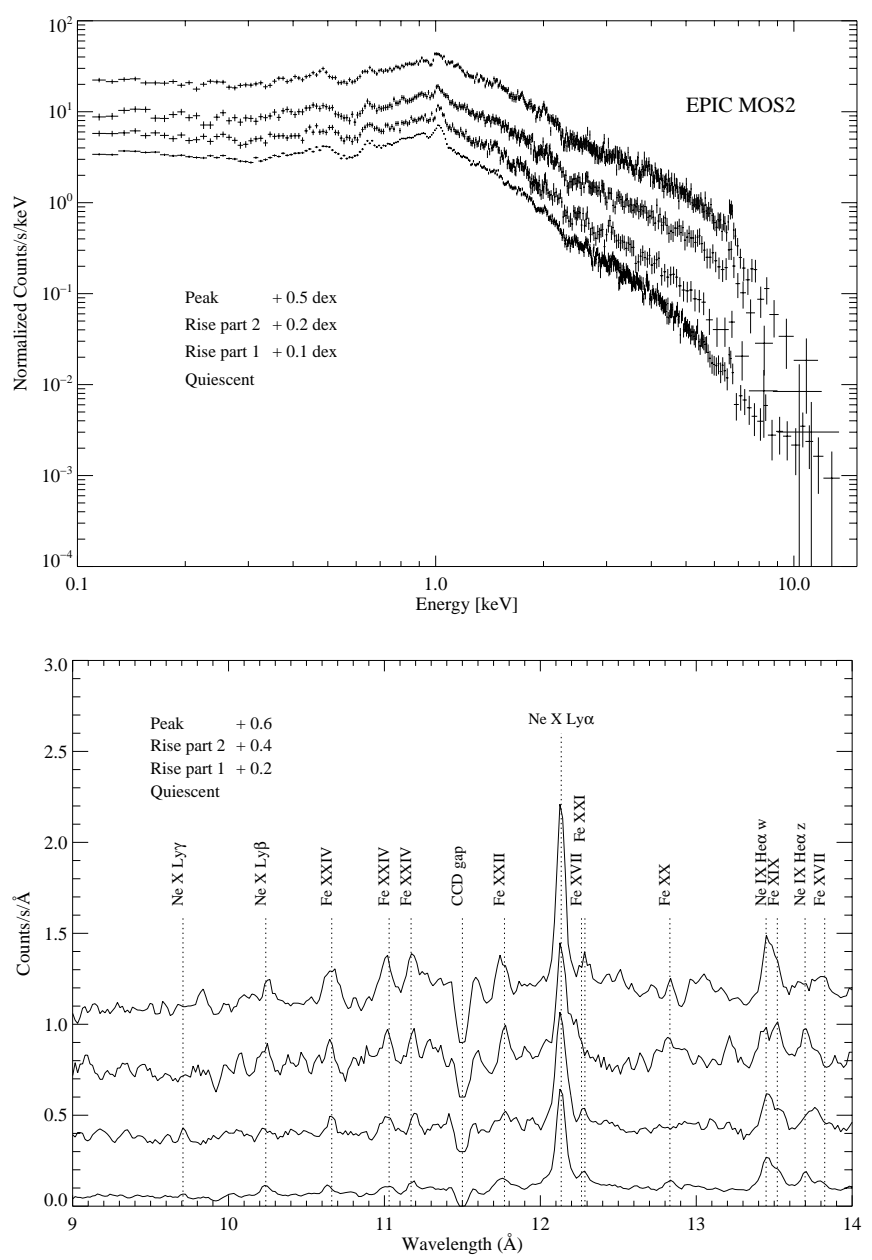

Fig. 3. Top: MOS2 spectra of the four time segments, from quiescent (lowest spectrum) to flare peak (uppermost spectrum). Spectra are shifted in flux as indicated, for illustration. Bottom: extract from smoothed RGS spectra of the four time segments (lowest: quiescent; uppermost: flare peak). Note relative shifts of the spectra

\subsection{Time-dependent spectroscopy}

Table 3 gives best-fit results for the time-dependent spectral analysis of RGS2 data. Although the detailed description of the response of both spectrometers is expected to evolve over time, we have used RGS2 for the present analysis as this description is more advanced than for RGS1. Similar elemental abundance variations and a similar temperature structure are, however, also observed in RGS1.

We have fitted the quiescent RGS2 spectrum with collisional ionization equilibrium (CIE) models in the Utrecht software SPEX 2.0 (Kaastra et al. 1996). In order to obtain an optimum description of the thermal structure and to determine, at the same time, the elemental abundances, we used as many components as possible and necessary to obtain a good fit to the data. We found that four components lead to good results. The $\chi^{2}$ procedure did not require a fifth temperature component, and three or fewer CIE components were insufficient. For the flare parts, the quiescent best-fit model was frozen and an additional CIE 
Table 3. Best fit to the time-dependent RGS2 spectra with $90 \%$ confidence ranges for a single parameter. "..." entries mean that the value was kept fixed to its quiescent value (see text for details). Abundances are relative to solar photospheric values (Anders \& Grevesse 1989)

\begin{tabular}{lllll}
\hline Parameter & $Q$ & $R 1^{\mathrm{a}}$ & $R 2^{\mathrm{a}}$ & $P^{\mathrm{a}}$ \\
\hline$k T_{1}[\mathrm{keV}]$ & $0.29_{-0.06}^{+0.17}$ & $\ldots$ & $\ldots$ & $\ldots$ \\
$k T_{2}[\mathrm{keV}]$ & $0.65_{-1.1}^{+0.04}$ & $\ldots$ & $\ldots$ & $\ldots$ \\
$k T_{3}[\mathrm{keV}]$ & $1.24_{-0.20}^{+0.16}$ & $\ldots$ & $\ldots$ & $\ldots$ \\
$k T_{4}[\mathrm{keV}]$ & $2.58_{-0.77}^{+2.7}$ & $\ldots$ & $\ldots$ & $\ldots$ \\
$k T_{5}[\mathrm{keV}]$ & & $1.20_{-0.25}^{+0.60}$ & $2.50_{-0.51}^{+0.88}$ & $3.12_{-0.57}^{+0.96}$ \\
$\log E M_{1}\left[\mathrm{~cm}^{-3}\right]$ & $52.32_{-0.32}^{+0.38}$ & $\ldots$ & $\ldots$ & $\ldots$ \\
$\log E M_{2}\left[\mathrm{~cm}^{-3}\right]$ & $53.38_{-0.07}^{+0.06}$ & $\ldots$ & $\ldots$ & $\ldots$ \\
$\log E M_{3}\left[\mathrm{~cm}^{-3}\right]$ & $53.42_{-0.30}^{+0.16}$ & $\ldots$ & $\ldots$ & $\ldots$ \\
$\log E M_{4}\left[\mathrm{~cm}^{-3}\right]$ & $53.41_{-0.31}^{+0.19}$ & $\ldots$ & $\ldots$ & $\ldots$ \\
$\log E M_{5}\left[\mathrm{~cm}^{-3}\right]$ & & $53.51_{-0.08}^{+0.07}$ & $54.00_{-0.03}^{+0.03}$ & $54.22_{-0.02}^{+0.02}$ \\
$\mathrm{C}$ & $0.38_{-0.08}^{+0.08}$ & $\ldots$ & $\ldots$ & $\ldots$ \\
$\mathrm{N}$ & $0.60_{-0.54}^{+0.55}$ & $\ldots$ & $\ldots$ & $\ldots$ \\
$\mathrm{O}$ & $0.35_{-0.03}^{+0.03}$ & $0.03_{-0.03}^{+0.15}$ & $\ldots$ & $0.46_{-0.13}^{+0.19}$ \\
$\mathrm{Ne}$ & $0.93_{-0.09}^{+0.10}$ & $0.46_{-0.45}^{+0.69}$ & $\ldots$ & $1.32_{-0.44}^{+0.62}$ \\
$\mathrm{Mg}$ & $0.10_{-0.07}^{+0.07}$ & $\ldots$ & $\ldots$ & $0.44_{-0.44}^{+0.66}$ \\
$\mathrm{Si}$ & $0.18_{-0.12}^{+0.12}$ & $1.02_{-1.02}^{+1.28}$ & $1.56_{-1.30}^{+1.45}$ & $1.94_{-0.78}^{+0.97}$ \\
$\mathrm{~S}$ & $0.07_{-0.07}^{+0.05}$ & $\ldots$ & $\ldots$ & $\ldots$ \\
$\mathrm{Ar}$ & $1.23_{-0.56}^{+0.63}$ & $\ldots$ & $\ldots$ & $\ldots$ \\
$\mathrm{Ca}$ & $0.10_{-0.10}^{+0.19}$ & $\ldots$ & $\ldots$ & $\ldots$ \\
$\mathrm{Fe}$ & $0.15_{-0.02}^{+0.02}$ & $0.09_{-0.06}^{+0.12}$ & $0.32_{-0.13}^{+0.20}$ & $0.40_{-0.11}^{+0.19}$ \\
$\mathrm{Ni}$ & $0.06_{-0.06}^{+0.10}$ & $\ldots$ & $\ldots$ & $\ldots$ \\
\hline
\end{tabular}

${ }^{a}$ Entries related to the free CIE model (see text).

component was added to describe the flare plasma. We refrain from fitting the MOS data in terms of a full coronal model with variable abundances at this time, since the combination of its still improving calibration with considerable optical contamination and residual X-ray pile-up due to the brightness of HR 1099 (both optically and in X-rays) may bias the results. We obtained, however, rough fitting estimates for the emission measures and temperatures of the hotter components - see below. Figure 3 shows an extract of the RGS2 and the EPIC MOS2 spectra for the four time intervals. Signatures of heating are evident: the high- $T$ tail of the CCD spectra flattens significantly during the flare, a direct sign of a temperature increase. Furthermore, the RGS spectra show that the Fe XxIV lines increase in intensity, relative to the underlying continuum.

Several elements that show lines in the RGS band had their abundances (relative to the solar photospheric values given by Anders \& Grevesse 1989) left free to vary in the fitting process. In the quiescent model, the abundance of each element is kept at the same value in all three CIE components. For the flare parts, the abundances of the free CIE model were independent of the quiescent abundances. Several free elements did not improve the fit, and therefore their abundances were kept fixed at their quiescent values. During the flare, the elemental abundances were difficult to derive due to the low signal-to-noise $(S / N)$ ratio. Nevertheless, Fe and $\mathrm{Si}$ abundances appear to

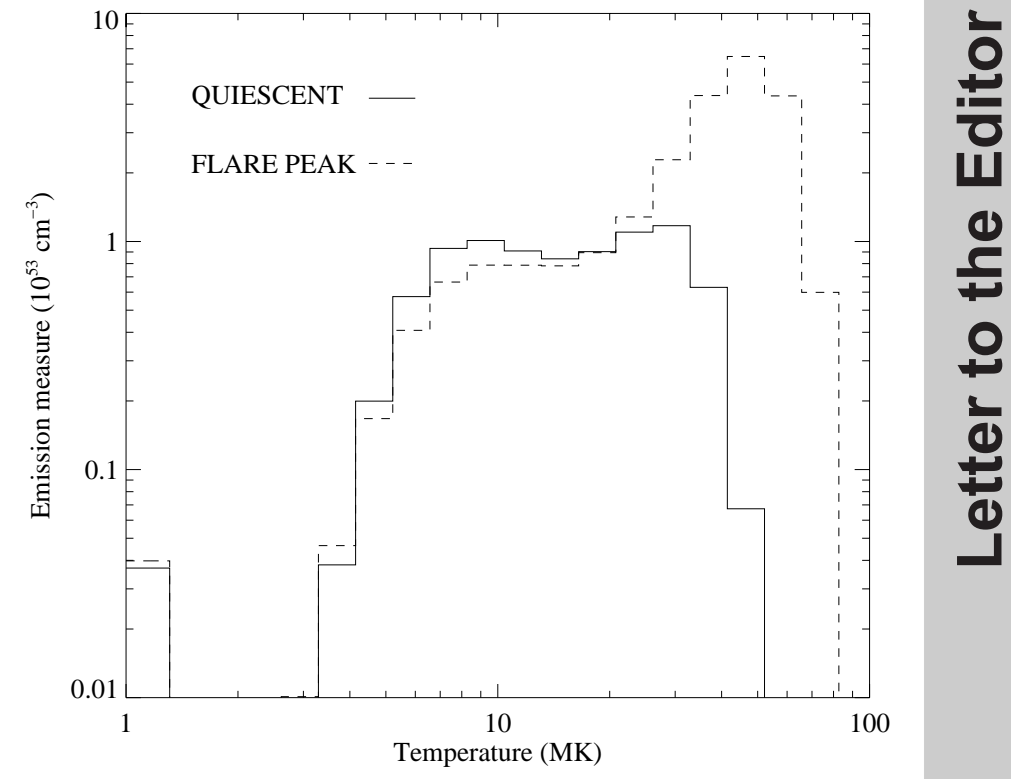

Fig. 4. Realizations of the quiescent (solid) and flare peak (dash) EM distributions using Chebychev polynomials of order 6

increase significantly (although with large 90\% confidence ranges). The RGS2 data determine an increase of temperature from $1.2 \mathrm{keV}$ to $3.1 \mathrm{keV}$. From the MOS2 data, we tentatively derive a high-temperature component of 2.8 , $4.4,8.0,7.2 \mathrm{keV}$ in the quiescent, flare rise (parts 1 \& 2), and flare peak intervals, respectively. These temperatures do not contradict the apparently lower $T$ determined by the RGS2. At such temperatures, there are too few spectroscopic features in the RGS band to discriminate between different temperatures, and small residual calibration uncertainties become important. For flare studies, combining the RGS (for elemental abundance studies and $T \leq 2 \mathrm{keV}$ ) with the EPICs (to constrain high- $T$ tails) is ideal. We also note that the MOS2 data support the view that the $\mathrm{Fe}$ and $\mathrm{Si}$ abundances increase, with values consistent with RGS2 results. But note also that the high-FIP element Ne appears to have an abundance that does not vary beyond the significance limits.

Using abundances from the multi-CIE fits, EM distributions have been derived from RGS spectra. Figure 4 shows realizations of the $E M$ distribution for the quiescent and flare peak spectra. Consistent with the multiCIE approach, the quiescent $E M$ distribution spans over a decade in temperature, with significant emission measure from $5 \mathrm{MK}$ to $30 \mathrm{MK}$. Note that the presence of $E M$ around $3-5 \mathrm{MK}$ is also significant and mainly necessary to fit the bright O VII He-like triplet around $22 \AA$ and the $\mathrm{C}$ VI Ly $\alpha$ line at $33.7 \AA$. The flare $E M$ distribution displays a gradual increase in the upper temperature. Fits for the flare peak (Fig. 4) show that a very hot EM $(>30 \mathrm{MK})$ dominates the emission. During the flare, the cooler $E M(T<20 \mathrm{MK})$ is consistent with the quiescent distribution. The quiescent density derived from the O VII triplet is low, with $n_{\mathrm{e}}<10^{10} \mathrm{~cm}^{-3}$ for the cooler material. 
During the flare, despite the poor $S / N$, the data suggest that the density of this relatively cool material does not increase. This behaviour is consistent with the absence of detection of a density increase in the cool plasma during flares found on AB Dor (Güdel et al. 2001).

\section{Discussion and conclusions}

A part of the "first light" observations of XMM-Newton has been analyzed, concentrating on overall light-curve variability and on the large flare at the end of the observation.

We found considerable variability over the $25 \mathrm{~d}$ observing time span. The X-ray flux varies with the binary phase, with the X-ray minimum at $\phi=0.5$, and maximum flux at $\phi=0$. A similar result was previously described by Agrawal \& Vaidya (1988) and Drake et al. (1994). In Chandra HETG data, Ayres et al. (2001) reported the detection of Nex line shifts consistent with the orbital motion of the active K1 IV star. Additionally, optical light curves showed, via Doppler imagery, the presence of a long-lived ( $\approx 11 \mathrm{yr}$ ) polar spot on HR 1099, together with transient $(<1 \mathrm{yr})$ low-latitude spots on the surface of the active K star (Vogt et al. 1999). Taken together, these observations suggest that one or several active regions are present on the hemisphere of the $\mathrm{K} 1$ star that is facing away from the G5 star. Since HR 1099 shows this effect consistently over years, it must be related to a fundamental pattern in its magnetic activity. It appears that the tidally locked rotation of the $\mathrm{K}$ star alters the internal dynamo in such a way that strong activity on the hemisphere facing the G star is suppressed. This, in turn, does not support the hypothesis that much of the X-ray emission in RS CVn binaries originates from the interbinary space (Siarkowski et al. 1996).

The maximum flux is not identical at every conjunction of the $\mathrm{K}$ star, consistent with a variable quiescent flux level coming from active regions. Note that it is difficult to distinguish between flares and rotationally modulated strong active regions in cases such as the wave at MJD 51582. Indeed, the maximum flux there is similar to the flare peak flux. Hardness analysis, however, clearly sets the flare at MJD 51593 apart from any other event during all other observations. On the other hand, EUV light curves from HR 1099 showed that large flares can have durations of one to two days (Osten \& Brown 1999), perhaps suggesting that the event at MJD 51582 is a slowly evolving but rotationally modulated flare. Conversely, it can be argued that the large flare at MJD 51593 is rotationally modulated as well, but given its restricted coverage, there is no clear evidence for geometric modulation.

Time-dependent spectroscopy of the large flare allowed us to derive time-dependent $E M$ distributions and abundances. The $E M$ reconstructions show a broad distribution in temperature, consistent with previous reports of broad hot $E M$ distributions for RS CVn systems (e.g., Griffiths \& Jordan 1998; Güdel et al 1999). The flare $E M$ peaks around $50-60 \mathrm{MK}$; the quiescent $E M$ seems to remain present during the flares, at least below $30 \mathrm{MK}$, indicating that the flare does not affect a very large fraction of the plasma in the visible active regions (see also Güdel et al. 1999). Audard et al. (2001) discuss the influence of potential inaccuracies in the atomic data utilized in the current version of SPEX on spectral fitting of RGS data. Especially, caution is in order for the $\mathrm{S}$ abundance. During the flare, Fe and Si (low-FIP; possibly S from MOS2 data) abundances appear to increase significantly, while the Ne (high-FIP) abundance stays at a value consistent with its quiescent value, within the error limits. The increase of low-FIP elemental abundances during the flare when highFIP elemental abundances stay at quiescent values can be interpreted as a direct signature of a FIP effect during flares. The quiescent abundances, however, are not compatible with a FIP effect. First, we find that all quiescent abundances are lower than solar photospheric abundances, in contrast to solar coronal behaviour (Feldman et al. 1992). Second, the low-FIP elements generally show lower abundances than the high-FIP elements. This "inverse FIP" effect was noted by Brinkman et al. (2001) and studied in detail for the higher- $S / N$ time-averaged RGS spectrum of HR 1099 that comprises all observations shown in Fig. 1.

Taken together, these observations seem to contradict the model that "quiescent" coronae are heated by a multitude of unresolved flares, since the FIP effect during the large flare counteracts trends in quiescent abundances. However, the solar case has shown a much larger complexity. There are several types of flares that show deviations from the standard picture. Schmelz (1993) reported on a class of flares that are selectively Ne rich. Brinkman et al. (2001) show that Ne is strongly overabundant with respect to $\mathrm{O}$, and they suggest that there are flare-like mechanisms different from very large flares that bring material into the corona, with a composition compatible with observations during quiescence. In any case, additional systematic studies using high-resolution spectroscopy will address this problem further.

Acknowledgements. We thank the referee, T. Montmerle, for his useful comments, and for his promptness in providing his report. M.A. acknowledges support from the Swiss National Science Foundation (grants 2100-049343 and 2000-058827), from the Swiss Academy of Sciences and from the Swiss Commission for Space Research. The Space Research Organization Netherlands (SRON) is supported financially by NWO.

\section{References}

Agrawal, P. C., \& Vaidya, J. 1988, MNRAS, 235, 239

Anders, E., \& Grevesse, N. 1989, Geochim. Cosmochim. Acta, 53, 197

Antonucci, E., Gabriel, A. H., \& Dennis, B. R. 1984, ApJ, 287, 917

Audard, M., Behar, E., Güdel, M., et al. 2001, A\&A, 365, L329 
Ayres, T. R., Brown, A., Osten, R. A., et al. 2001, ApJ (in press)

Bopp, W., \& Fekel, F. C. 1976, AJ, 81, 771

Brinkman, A. C., Behar, E., Güdel, M., et al. 2001, A\&A, 365, L324

Dennis, B. R. 1985, Sol. Phys., 100, 465

Drake, J. J., \& Kashyap, V. L. 1998, in ASP Conf. Ser. 154, Tenth Cambridge Workshop on Cool Stars, Stellar Systems, and the Sun, ed. R. A. Donahue, \& J. A. Bookbinder (San Francisco: ASP), 1014

Drake, J. J., Brown, A., Patterer, R. J., et al. 1994, ApJ, 421, L43

Drake, J. J., Laming, J. M., \& Widing, K. G. 1995, ApJ, 443, 393

Drake, J. J., Laming, J. M., \& Widing, K. G. 1997, ApJ, 478, 403

Favata, F., Reale, F., Micela, G., et al. 2000, A\&A, 353, 987

Fekel, F. C. 1983, ApJ, 268, 274

Feldman, U., Mandelbaum, P., Seely, J. F., Doschek, G. A., \& Gursky, H. 1992, ApJS, 81, 387

Griffiths, N. W., \& Jordan, C. 1998, ApJ, 497, 883

Güdel, M., Audard, M., Briggs, K., et al. 2001, A\&A, 365, L336

Güdel, M., Linsky, J. L., Brown, A., \& Nagase, F. 1999, ApJ, 511, 405

den Herder, J. W., Brinkman, A. C., Kahn, S. M., et al. 2001, A\&A, 365, L7

Jansen, F., Lumb, D., Altieri, B., et al. 2001, A\&A, 365, L1

Kaastra, J. S., Mewe, R., \& Nieuwenhuijzen, H. 1996, in UV and X-ray Spectroscopy of Astrophysical and Laboratory, ed. K. Yamashita, \& T. Watanabe (Tokyo: Univ. Acad. Press), 411

Lumb, D. H., Gondoin, Ph., Turner, M. J. L., et al. 2000, in SPIE Proceedings, vol. 4140 (in press)
Majer, P., Schmitt, J. H. M. M., Golub, L., Harnden, F. R., \& Rosner, R. 1986, ApJ, 300, 360

Mason, K. O., Breeveld, A., Much, R., et al. 2001, A\&A, 365, L36

Mewe, R. 1999, in X-ray Spectroscopy in Astrophysics, EADN School X., ed. J. van Paradijs, \& J. A. M. Bleeker (Berlin: Springer), 109

Osten, R. A., \& Brown, A. 1999, ApJ, 515, 746

Ottmann, R., \& Schmitt, J. H. M. M. 1996, A\&A, 307, 813

Pallavicini, R., \& Tagliaferri, G. 1998, Nucl. Phys. B, Proc. Suppl., 69, 29

Pasquini, L., Schmitt, J. H. M. M., \& Pallavicini, R. 1989, A\&A, 226, 225

Perryman, M. A. C., Lindegren, L., Kovalevsky, J., et al. 1997, A\&A, 323, L49

Randich, S., Giampapa, M. S., \& Pallavicini, R. 1994, A\&A, 283,893

Schmelz, J. T. 1993, ApJ, 408, 373

Schmitt, J. H. M. M., Collura, A., Sciortino, S., et al. 1990, ApJ, 365, L704

Schmitt, J. H. M. M., Stern, R. A., Drake, J. J., \& Kürster, M. 1996, ApJ, 464, 898

Siarkowski, M., Preś, P., Drake, S. A., White, N. E., \& Singh, K. P. 1996, ApJ, 473, 470

Strüder, L., Briel, U. G., Dennerl, K., et al. 2001, A\&A, 365, L18

Tsuboi, Y., Koyama, K., Murakami, H., et al. 1998, ApJ, 503, 894

Turner, M. J. L., Abbey, A., Arnaud, M., et al. 2001, A\&A, 365, L27

Vogt, S. S., Hatzes, A. P., Misch, A. A., \& Kürster, M. 1999, ApJS, 121, 547 